\title{
TRIGONOMETRY ON THE UNIT BALL OF A COMPLEX HILBERT SPACE
}

\author{
BY KYONG T. HAHN
}

Communicated by François Treves, September 25, 1974

1. Introduction. The unit disc furnished with the Poincare metric provides a model (the Poincaré model) for the hyperbolic geometry. The linear fractional transformations of the unit disc onto itself constitute the group of motions. An analogous phenomenon arises in the unit ball of any complex Hilbert space when it is furnished with the Carathéodory-Reiffen metric.

The purpose of this note is to announce the hyperbolic version of the laws of sines and cosines, and the Pythagorean theorem on the unit ball of any complex Hilbert space.

2. Preliminaries. Let $X$ and $Y$ be complex Banach spaces and let $D \subset X$ be a domain (= open connected subset of $X$ ). A map $f: D \rightarrow Y$ is holomorphic if the Fréchet derivative of $f$ at each $x \in D$ (denoted by $D f(x)$ ) exists and is complex linear. Let $G$ be a domain of $Y$. A map $f: D \rightarrow G$ is biholomorphic if the inverse map $f^{-1}: G \rightarrow D$ exists and both $f$ and $f^{-1}$ are holomorphic. A domain $D$ is homogeneous if for each pair of points $x, x^{\prime}$ in $D$ there exists a biholomorphic map $f: D \rightarrow D$ with $f(x)=x^{\prime}$.

Let $\Delta(D)$ denote the class of holomorphic maps of $D$ into the unit disc $\Delta$ in the complex plane $C$. Following [1], we define the CarathéodoryReiffen metric by

$$
\alpha_{D}(x, \xi)=\sup \{|D f(x) \xi|: f \in \Delta(D)\}, \quad x \in D, \quad \xi \in X,
$$

where || denotes the norm in $C$.

The distance $\rho_{D}\left(x, x^{\prime}\right)$ between two points $x$ and $x^{\prime}$ in $D$ is defined in the usual way. Namely,

AMS (MOS) subject classifications (1970). Primary 50A10, 46A20; Secondary $58 \mathrm{~B} 20,58 \mathrm{C} 20$.

Key words and phrases. Complex Hilbert space, hyperbolic geometry, CarathéodoryReiffen metric, laws of sines and cosines, Pythagorean theorem, geodesic triangle. 


$$
\rho_{D}\left(x, x^{\prime}\right)=\inf \int_{a}^{b} \alpha_{D}(\gamma(t), D \gamma(t) d t)
$$

where the infimum runs over all piecewise continuously differentiable curves $\gamma:[a, b] \rightarrow D$ with $\gamma(a)=x, \gamma(b)=x^{\prime}$.

Consider the unit ball $B$ in the complex Hilbert space $H$ furnished with the metric $\alpha_{B}$. For any pair of distinct points $x$ and $y$ in $B$ there exists a unique shortest "geodesic" curve joining them. Indeed, it is easy to see that for any nonzero vector $a \in B$ the straight line segment $\sigma[0, a]: \gamma(t)$ $=t a, t \in[0,1]$, is the unique shortest geodesic between 0 and $a$, and the length of $\sigma[0, a]$ is given by $\rho_{B}(0, a)=\tanh ^{-1}\|a\|$.

The same method used in the proof of Lemma 4.1 of [2] also works in this case. The shortest geodesic between any two points $a$ and $b$ in $B$ is then determined uniquely by the image curve of the straight line segment $\sigma\left[0, T_{-a}(b)\right]$ under $y=T_{a}(x)$. Therefore, any given three points in $B$ determine a "geodesic triangle" uniquely. A geodesic triangle consists of three sides given by the shortest geodesics and three angles defined as follows.

First we define the inner product of two vectors $\xi, \eta$ of $H$ at $x \in B$ by

$$
\alpha_{B}(x ; \xi, \eta)=\alpha_{B}\left(0 ; D T_{-x}(x) \xi, D T_{-x}(x) \eta\right)
$$

while the inner product of $\xi, \eta$ at $0 \in B$ is given by $\alpha_{B}(0 ; \xi, \eta)=(\xi, \eta)$.

As usual, the angle $H$ between $\xi$ and $\eta$ at $x \in B$ is defined by

$$
\cos H=\left|\alpha_{B}(x ; \xi, \eta)\right| / \alpha_{B}(x, \xi) \alpha_{B}(x, \eta), \quad 0 \leqslant H \leqslant \pi / 2 .
$$

We agree to call the angle $\theta$ defined by $\cos \theta=|(\xi, \eta)| /\|\xi\|\|\eta\|$ the "Hilbert space angle" between $\xi$ and $\eta$. In particular, if $x=0, H=\theta$. Therefore, for each pair of vectors $\xi$ and $\eta$ in $H$ and each $x \in B$, there correspond two angles which coincide at $0 \in B$.

3. Main results. We now state the main theorems.

THEOREM 1 (LAW OF SINES). Let $a_{1}, a_{2}$ and $a_{3}$ be the vertices of $a$ geodesic triangle $\Delta$ in $B$ whose angle at $a_{i}$ is $H_{1}$. Let $g_{i}$ be the lengths of the three sides of $\Delta$ opposite to $H_{i}, i=1,2,3$. Then

$$
\frac{\sin H_{1}}{\sinh g_{1}}=\frac{\sin H_{2}}{\sinh g_{2}}=\frac{\sin H_{3}}{\sinh g_{3}} \text {. }
$$


THEOREM 2 (LAW OF COSINES). Let $\Delta$ be a geodesic triangle in $B$ given as in Theorem 1. Then

$$
\cosh g_{3}=\cosh g_{1} \cosh g_{2}-\sinh g_{1} \sinh g_{2} \cos H_{3}
$$

holds if and only if $\operatorname{Im}\left(T_{-a_{3}}\left(a_{1}\right), T_{-a_{3}}\left(a_{2}\right)\right)=0$.

In particular, we have

Theorem 3 (Pythagorean theorem). Let $\Delta$ be a geodesic triangle in $B$ given as in Theorem 1. Then

$$
\cosh g_{3}=\cosh g_{1} \cosh g_{2}
$$

holds if and only if $\mathrm{H}_{3}=\pi / 2$.

The proofs of the above theorems depend on the invariant property of the metric $\alpha_{B}$ under the möbius transformations of $B$, the homogeneity of $B$ and the following

Lemma. Let $H$ be the angle between two vectors $\xi$ and $\eta$ in $H$ at $x \in B$ relative to $\alpha_{B}$ and let $\theta$ be the corresponding Hilbert space angle. If.either $\xi$ or $\eta$ is expressed by a complex multiple of $x$, then

$$
\left(\cos ^{2} \theta\right) / \cos ^{2} H=1-\|x\|^{2} \sin ^{2} \theta .
$$

The explicit forms of the möbius transformations of $B$ and the necessary computational machinary are provided in [4] and [5]. The corresponding results in the space $C^{n}$ are already obtained in [2] and [3].

Complete proofs of our results and details will appear elsewhere.

ACKNOWLEDGEMENT. The author takes this opportunity to acknowledge his indebtedness to Professor L. Harris for several helpful discussions concerning the problem.

\section{REFERENCES}

1. C. J. Earle and R. S. Hamilton, A fixed point theorem for holomorphic mappings, pings, Global Analysis, Proc. Sympos. Pure Math., vol. 16, Amer. Math. Soc., Providence, R. I., 1965.

2. K. T. Hahn, The non-euclidean Pythagorean theorem with respect to the Bergman metric, Duke Math. J. 33 (1966), 523-534. MR 34 \#6149.

3. - Trigonometry in a hyperbolic space, Duke Math. J. 35 (1968), 739745. MR 37 \#5826. 
4. L. A. Harris, Schwarz's lemma and the maximum principle in infinite dimensional spaces, Thesis, Cornell University, Ithaca, N. Y., 1969.

5. - Bounded symmetric homogeneous domains in infinite dimensional spaces, Proc. Infinite Dimensional Holomorphy, Lecture Notes in Math., vol. 364, SpringerVerlag, Berlin and New York, 1973, 13-40.

DEPARTMENT OF MATHEMATICS, PENNSYLVANIA STATE UNIVERSITY, UNIVERSITY PARK, PENNSYLVANIA 16802 